\title{
Lactobacillus delbrueckii alleviates depression-like behavior through inhibiting toll-like receptor 4 (TLR4) signaling in mice
}

\author{
Xiangjie Qiu ${ }^{1}$, Guojun Wu ${ }^{1}$, Lili Wang ${ }^{1}$, Yurong $\operatorname{Tan}^{1}$, Zhi Song ${ }^{2}$ \\ ${ }^{1}$ Department of Medical Microbiology, Xiangya School of Medicine, Central South University, Changsha, China; ${ }^{2}$ Department of General Surgery, \\ The Third Xiangya Hospital, Central South University, Changsha, China \\ Contributions: (I) Conception and design: X Qiu, Y Tan; (II) Administrative support: L Wang; (III) Provision of study materials or patients: Z Song; (IV) \\ Collection and assembly of data: X Qiu, G Wu; (V) Data analysis and interpretation: X Qiu, Y Tan; (VI) Manuscript writing: All authors; (VII) Final \\ approval of manuscript: All authors. \\ Correspondence to: Yurong Tan. Department of Medical Microbiology, Xiangya School of Medicine, Central South University, Changsha 410078, \\ China. Email: yurongtan@csu.edu.cn; Zhi Song. Department of General Surgery, The third Xiangya Hospital, Central South University, Changsha \\ 410000, China. Email: songzhi200@qq.com.
}

Background: The intestinal flora can influence behavior through the microbiota-gut-brain axis and is closely related to the occurrence and development of nervous system diseases such as depression. Probiotics like Lactobacillus may regulate the balance of the intestinal flora and play an active role in preventing and treating depression.

Methods: Eight-week-old C57BL/6J mice (n=32) were randomly and equally divided into a normal control group, a control + Lac group, a model group, and a model + Lac group. The model and model + Lac groups were intraperitoneally injected with $1.2 \mathrm{mg} / \mathrm{kg}$ lipopolysaccharide for 7 days, and the behavior of the mice was assessed 24 hours later. The normal and model groups received intragastric administration of saline daily, while the control $+L a c$ and model $+L a c$ groups were given $10^{9}$ cfu $L a c$ intragastrically daily for 7 days. The inhibitory effect of $L a c$ and its fermentation products on depression-related bacteria were examined in vitro.

Results: $L a c$ effectively inhibited the production of depression-like behaviors in mice. The expression levels of zonula occludens-1 (ZO-1) and E-cadherin in the small intestine in the model group were significantly decreased, but Lac abrogated this effect. Overactivation of microglia and decreased expression of dopamine transporter (DAT) in brain tissues, which are closely related to depression, were also abrogated by Lac treatment. Furthermore, the expression of toll-like receptor 4 (TLR4) and nod-like receptor protein-3 (NLRP3), as well as the level of interleukin-1 beta (IL-1 $\beta$ ) in the intestine and brain, were all significantly increased; however, these effects were subsequently abrogated by Lac. Moreover, Lac inhibited dysbiosis through its metabolites.

Conclusions: $L a c$ has a remarkable antidepressant function, which it performs through the inhibition of dysbiosis (via its metabolites) and pattern recognition receptor TLR4 signaling.

Keywords: Depression; Lactobacillus delbrueckii subsp. bulgaricus; toll-like receptor 4 (TLR4); nod-like receptor protein-3 (NLRP3)

Submitted Jun 01, 2020. Accepted for publication Nov 27, 2020.

doi: 10.21037/atm-20-4411

View this article at: http://dx.doi.org/10.21037/atm-20-4411 


\section{Introduction}

Depression is one of the most common mental disorders in the world. As a mood disorder with many possible causes, depression is characterized by a significant and lasting depressed mood (1). Statistics reported by the World Health Organization show that more than 300 million people suffer from depression worldwide, and it was projected to become the second leading disease for morbidity and mortality by 2020 (2). The etiology and pathogenesis of depression are not very clear, but abnormalities in neural pathway function and structure and neuroendocrine function are known to be involved (3). Genetic factors and stressful life events are also closely related to depression (4). Studies have shown that alcohol abuse, pregnancy, social defeat, stress, and other factors can increase inflammatory cytokine release (5-7). Inflammation, directly and indirectly, affects the physiological and pathological process of depression through multiple pathways, including nerve, endocrine, and immune pathways (8). Various stresses induce microglia activation and induce the secretion of proinflammatory cytokines by cells, resulting in neuroinflammation and subsequent depression $(9,10)$. This study used lipopolysaccharide (LPS) to induce depression-like behavior in C57BL/6J mice. The characteristics of the LPS-induced neuroinflammatory model are extremely similar to those of depression and some neurodegenerative diseases, so it is widely used to study depression-related mechanisms $(11,12)$.

At present, antidepressants are the main treatment for depression. However, for $30-40 \%$ of patients, the treatment efficacy is poor (13), and most of the drugs are designed to interfere with the activity of neurotransmitters in the brain, resulting in headache, nausea, irritability, sedation, sexual dysfunction, and other side effects (14). When taken in appropriate amounts, probiotics such as Lactobacillus and Bifidobacterium have health-promoting effects, which they exert through the brain-gut axis via the neuroendocrine, nerve, and immune pathways (15). A recent study found that the number of Lactobacilli and Bifidobacteria in the intestinal tract of depressive patients was significantly decreased. In contrast, the number of Enterobacteriaceae and Enterococcus was significantly increased (16), suggesting that dysbiosis is present in the intestinal tract of depressive patients and that recovery of dysbiosis may be a novel approach to treating depression. Lactobacillus is the main type of probiotic, which can regulate the balance of the intestinal flora and play an active role in preventing and alleviating depression (17-21). Lactobacillus delbrueckii subsp. bulgaricus (Lac) is the most widely used probiotic in dairy products $(22,23)$.

To further explore Lactobacillus's components and mechanisms against depression-like symptoms, we studied the therapeutic effect of Lac on depression and its underlying mechanism by performing in vivo and in vitro experiments. We present the following article following the ARRIVE reporting checklist (available at http://dx.doi. org/10.21037/atm-20-4411).

\section{Methods}

Experiments were performed under a project license (NO.: 2017-S000) granted by the Institutional Ethics Committee of Xiangya School of Medicine, Central South University. All animal experiments were conducted in compliance with the national or institutional guidelines for the care and use of animals.

\section{Mice model}

Eight-week-old C57BL/6J mice purchased from Hunan Tianqin Biological Technology Co., Ltd. were selected. The mice were kept in a single cage under suitable light conditions and at a constant temperature of $25^{\circ} \mathrm{C}$ for 7 days. They were fed with standard laboratory chow consisting of $18 \%$ protein, $58 \%$ carbohydrate, $4 \%$ fat, $15 \%$ water, and $5 \%$ other ingredients, including minerals, vitamins, and salts (Hunan Tianqin Biological Technology Co., Ltd., Changsha, China). The feeding room was well ventilated, and drinking water was freely available.

After 7 days, the mice ( $\mathrm{n}=32)$ were divided into a normal control group (CG), a control + Lac group $(C G+L a c)$, a model group (LPS), and a treatment group (LPS + Lac) using a random number table ( $\mathrm{n}=8$ in each group). The number of animals needed for the paired test was deduced by observing when the $t$-test reached the significance level of 0.05 . The mice in the LPS and LPS + Lac groups were given LPS at a dose of $1.2 \mathrm{mg} / \mathrm{kg}$ (Escherichia coli 055 : B5, Sigma-Aldrich, MO, USA) via intraperitoneal injection for 7 days $(11,12)$. After 24 hours, the mice's behavior was assessed to determine whether the depression-like model had been successfully constructed. Then, the mice in the CG and LPS group were given saline by gavage every day, while the mice in the CG + Lac and LPS + Lac groups were given $10^{9}$ cfu of Lac by gavage every day. Seven days later, the mice in each group were tested. All animal experiments in this study conformed to the local principles for managing laboratory animals and followed the Guidelines for the 
Management and Use of Laboratory Animals published by the National Institutes of Health of the United States. Lac was kindly gifted by the College of Food Science and Technology, Hunan Agricultural University.

\section{Behavioral assessment}

Sugar-water preference test: firstly, the mice were adaptively fed $1 \%$ sucrose water for 48 hours. Then, after the mice had been deprived of water for 12 hours, a $1 \%$ sucrose bottle and a bottle of pure water were put in the cage. The consumption of sugar water and pure water was monitored for 4 hours. During the 4 hours, the sugar water and pure water bottles' positions were changed every 2 hours to prevent errors in the results caused by the mice's preference for drinking positions. The sugar water preference was calculated according to the formula: sugar water consumption $\%$ = sugar water consumption (g)/(sugar water consumption + pure water consumption) $(\mathrm{g}) \times 100 \%$.

Forced swimming test: the ambient and water temperatures were set at $25^{\circ} \mathrm{C}$. The mice were placed in a round glass jar with a height of $20 \mathrm{~cm}$, a depth of $10 \mathrm{~cm}$, and a diameter of $12 \mathrm{~cm}$. Immobility was recorded when the mice stopped struggling, floated, or kept their head floating on the surface of the water with only slight limb movements. Observations continued for 6 minutes, and the cumulative sum of immobility time during the final 4 minutes was recorded. The percentage of time spent immobile was calculated as follows: immobility time/total time $\times 100 \%$.

\section{Sample collection}

To obtain tissue from the small intestine $(2-3 \mathrm{~cm})$, the mice's abdominal cavity was exposed after ether anesthesia and rinsed with $0.9 \%$ sodium chloride solution. Formed excrement in the large intestine was collected. Hippocampal tissue was obtained by cutting open the skull after perfusion with $0.9 \%$ sodium chloride followed by $4 \%$ paraformaldehyde solution (24).

\section{Enzyme linked immunosorbent assay}

Small intestinal or hippocampal tissues $(100 \mathrm{mg})$ were washed in precooled phosphate buffer solution (PBS) (0.02 mol/L, $\mathrm{pH} 7.0-7.2)$ to remove blood. After weighing, the tissue was transferred into a glass homogenizer, and $5 \mathrm{~mL}$ of precooled PBS was added for each gram of sample, ground the tissue fully on ice, and then the homogenization solution was obtained using ultrasound. The prepared homogenate was centrifuged at 5,000 $\mathrm{g}$ for 5 minutes, and the supernatant was retained. Finally, $5 \mu \mathrm{L}$ of supernatant was taken, and the level of interleukin (IL)- $1 \beta$ was detected following the instructions of a mouse IL-1 $\beta$ ELISA (enzyme-linked immunosorbent assay) Kit (JL46166, Shanghai Jianglai Biotechnology Co., Ltd.).

\section{Real-time fuorescence quantitative PCR}

The primers of glyceraldehyde- 3 phosphate dehydrogenase $(G A P D H)$, zonula occludens-1 (ZO-1), E-cadherin, nodlike receptor protein-3 (NLRP3), toll-like receptor 4 (TLR4), dopamine transporter (DAT), and ionized calcium binding adaptor molecule-1 (Iba-1) genes (Table S1) were designed using the Primer Premier 5.0 software (Premier Biosoft International, Palo Alto, CA, USA). Total RNA was extracted from the small intestinal and hippocampal tissues with TRIzol, and RNA purity was analyzed using a nucleic acid analyzer. RNA $(1 \mu \mathrm{g})$ from each sample was used for reverse transcription (RT). Synthesis of cDNA was performed through RT (R312-01, Nanjing Vazyme Biotech Co., Ltd., Nanjing, China) and amplified using a quantitative polymerase chain reaction (qPCR) kit (Q511-02, Nanjing Vazyme Biotech Co., Ltd., Nanjing, China) according to the manufacturer's instructions. The reaction mixture consisted of the following: $1.5 \mu \mathrm{L}$ cDNA, $12.5 \mu \mathrm{L}$ SYBR Green qPCR Master Mix, $1.5 \mu \mathrm{L}$ each of the forward and reverse primers $(10 \mu \mathrm{M})$, and diethylpyrocarbonate water (total volume, $25 \mu \mathrm{L}$ ). The RT-PCR was performed under the following conditions: $94{ }^{\circ} \mathrm{C}$ for 3 minutes, 35 cycles of $94{ }^{\circ} \mathrm{C}$ for 45 seconds, $51^{\circ} \mathrm{C}$ for 45 seconds, and $72{ }^{\circ} \mathrm{C}$ for 45 seconds. The relative mRNA expression was calculated using the $2^{-\Delta \Delta \mathrm{Ct}}$ method (25).

Total DNA was extracted from the feces of mice in each group. The purity of DNA was analyzed using a nucleic acid analyzer. The primers (Lactobacillus N-acyltransferase: CGTTAGCCAGCCGACATT; ACGCATCAGGCAACAGCA, $129 \mathrm{bp}$ ) were used for qPCR under the following conditions: pre-denaturation at $94{ }^{\circ} \mathrm{C}$ for 3 minutes, denaturation at $94^{\circ} \mathrm{C}$ for 45 seconds, annealing at $51{ }^{\circ} \mathrm{C}$ for 45 seconds, prolongation at $72{ }^{\circ} \mathrm{C}$ for 45 seconds, and amplification of 65 cycles.

\section{Western blot}

Small amounts of small intestinal tissues and hippocampal 
tissues were ultrasonically lysed using $500 \mu \mathrm{L}$ of strong RIPA lysate containing phenylmethyl sulfonyl fluoride (PMSF). Total protein was collected by centrifuging at $12,000 \mathrm{rpm}$ at $0{ }^{\circ} \mathrm{C}$ for 25 minutes, and the protein concentrations were detected with a BCA protein assay kit (PA-115, Beijing Tiangen Biochemical Technology Co., Ltd.). Western blot (WB) was carried out to detect the protein expression of NLRP3, TLR4, Iba-1, and DAT with GAPDH used as an internal control. The primary antibodies (1:500) were produced by Abcam company (ab8245, ab178846, ab221845, ab214185, ab13556), and the secondary antibody (1:500) was produced by KPL company (074-1506, 074-1806) (26).

\section{Hematoxylin-eosin (H\&E) staining}

Resected small intestinal tissues were fixed with $10 \%$ formaldehyde solution for 48 hours, embedded in paraffin, and cut into 4- $\mu \mathrm{m}$-thick sections. The tissue sections were heated to $60{ }^{\circ} \mathrm{C}$ for 2 hours, dewaxed by xylene (twice, 15 minutes each time), and dehydrated using an increasing alcohol gradient $(75 \%, 95 \%$, and $100 \%)$ for 5 minutes each time. H\&E staining was performed, and the specimens were observed for pathological morphology under a light microscope (23).

\section{Analysis of the inbibitory effect of Lac and its fermentation products on depression-related bacteria}

Escherichia coli (6 strains), Pseudomonas aeruginosa (5 strains), Klebsiella pneumoniae subsp. Pneumonoiae (6 strains), Klebsiella oxytoca (6 strains), Serratia fonticola (4 strains), Serratia marcescens subsp. Marcescens (5 strains), Enterobacter aerogenes (5 strains), Acinetobacter baumannii (7 strains), Citrobacter freundii (4 strains), Enterobacter cloacae (E. cloacae) (5 strains), Stenotrophomonas maltophilia (6 strains), Staphylococcus aureus [8 strains, including 3 strains of methicillinresistant Staphylococcus aureus (MRSA)], and Staphylococcus epidermidis (6 strains) were isolated from the intestine. The nutrient broth and agar were products of Hangzhou Microbial Reagent Co., Ltd.; lactic acid bacteria culture medium (MRS) nutrient broth and agar were purchased from Hangzhou Baisi Biotechnology Co., Ltd. The tested bacterium and $L a c$ were mixed to proportions of 1:1, 1:2, $1: 3,1: 4$, and $1: 5$ and seeded in the most suitable mixed cultural medium.

The target bacteria were diluted to $1.5 \times 10^{8} \mathrm{cfu} / \mathrm{mL}$ with cultural medium, and $100 \mu \mathrm{L}$ of the bacterial suspension was coated onto the nutrient agar plate. Then, 5 Oxford cups (numbered 1, 2, 3, 4, and 5, respectively) were placed on the plate. No. 1 contained 48 -hour fermentation products of Lac with bacteria $(200 \mu \mathrm{L})$; No. 2 contained the bacteria-free supernatant of 48-hour fermentation products of $L a c(200 \mu \mathrm{L})$; No. 3 contained the bacteriafree supernatant of 48-hour fermentation products of $\mathrm{Lac}$ with the $\mathrm{pH}$ value adjusted to $7.2(200 \mu \mathrm{L})$; No. 4 contained lactic acid solution $(200 \mu \mathrm{L} ; \mathrm{pH}=4.0)$; and No. 5 (control group) contained MRS $(200 \mu \mathrm{L})$. Finally, the results were observed after incubation at $37^{\circ} \mathrm{C}$ for 18 to 24 hours.

\section{Statistical analysis}

SPSS 21.0 software was used to perform statistical analyses. Data were expressed as mean \pm standard deviation (SD). $T$-test was used for comparisons between two groups, two-way analysis of variance (ANOVA) was used for comparisons among multiple groups, and least significant difference (LSD) was used as a post-hoc test. The inhibition zone diameter was expressed in millimeters $(\mathrm{mm})$, and the experimental data of bacterial culture in vitro were expressed in terms of mean $\pm \mathrm{SD}$. Statistical significance was indicated by $\mathrm{P}<0.05$.

\section{Results}

\section{Lac effectively inbibited the occurrence of depression-like behavior in vivo}

The mice's body weights in each group were recorded, and no differences were found among the groups. However, the mice in the model group displayed a dull hair and decreased activity. None of the mice in this study died. All of the mice were included in each test and statistical analysis. To study the mechanism of Lac on depression-like symptoms, we conducted a sugar-water preference test. We found that the percentage of sugar water consumed in the model group was significantly lower than that in the control group, which was increased by Lac treatment (Figure 1A). The immobility time in the model group was significantly longer than that in the control group. Lac treatment was found to decrease the immobility time (Figure $1 B$ ), indicating that it could significantly inhibit the occurrence of depressionlike behavior in mice. Correspondingly, the number of $L a c$ in the feces of the mice in the model group was significantly decreased compared to that in the control group, and it was increased significantly in the treatment group compared to 
A

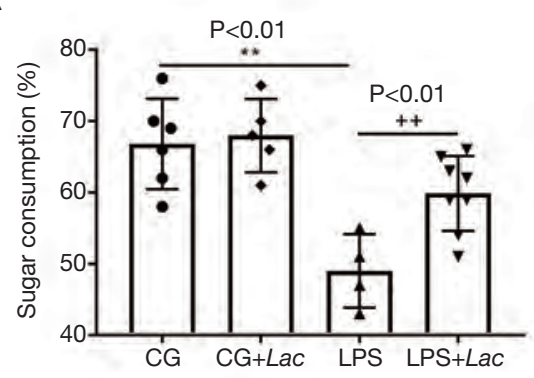

D

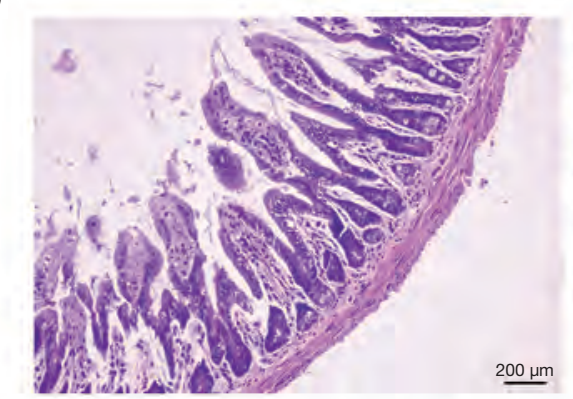

CG

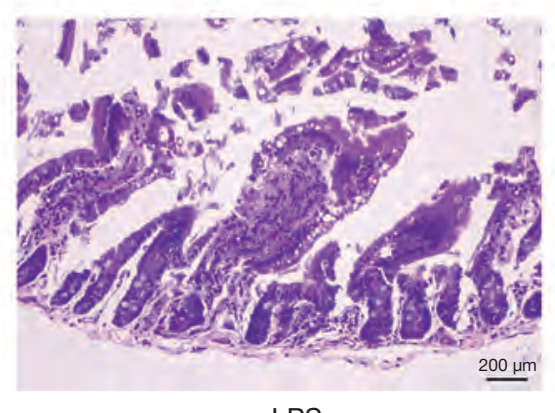

LPS
B
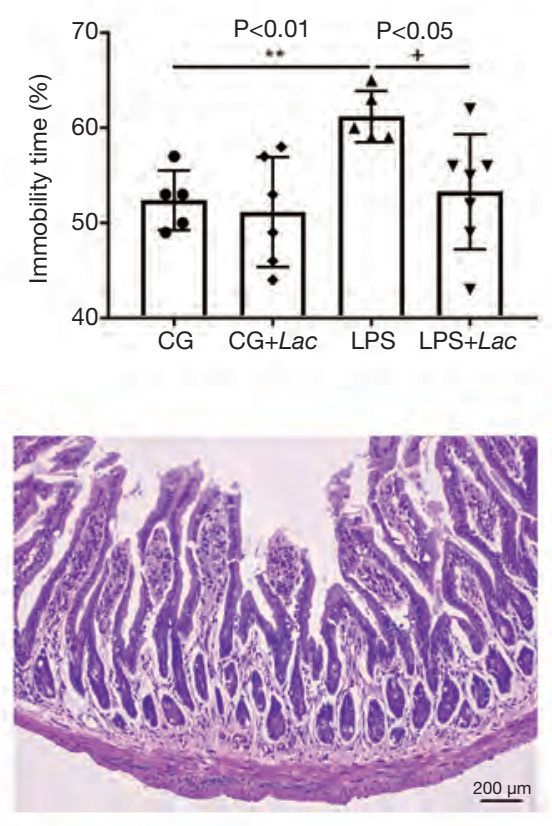

Lac

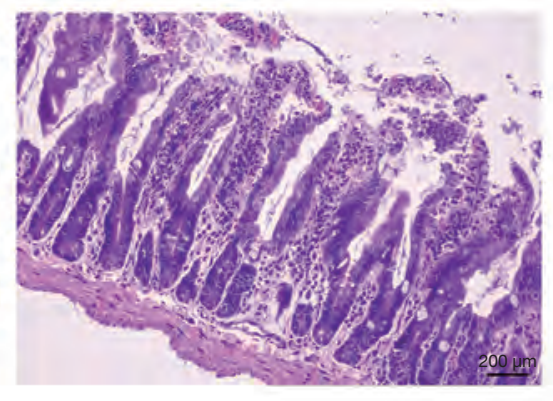

LPS + Lac
C

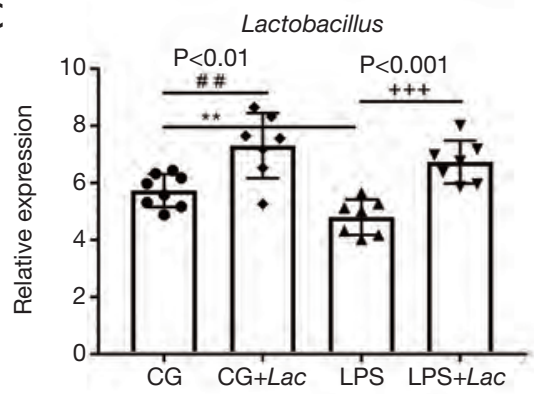

E

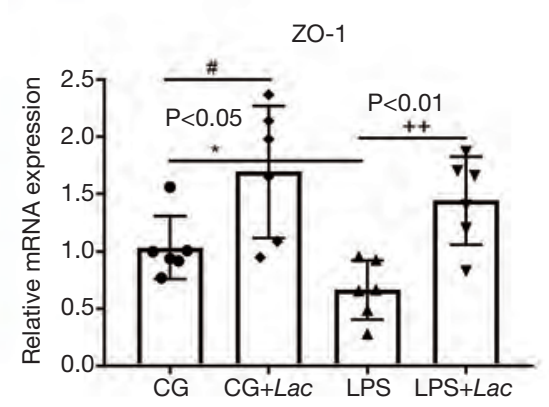

$\mathrm{F}$

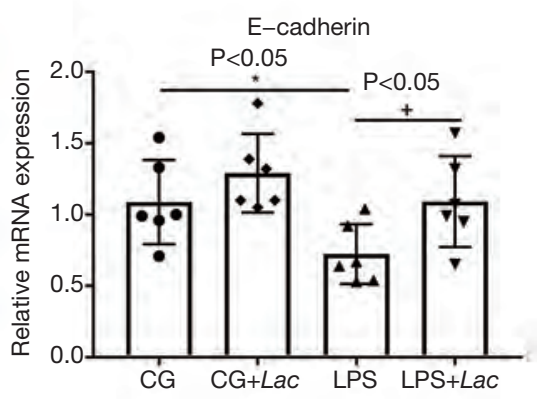

Figure 1 Lac effectively inhibited the occurrence of depression-like behavior in vivo (n=8). (A) The percentage of sugar consumption; (B) percentage of time spent immobile; (C) quantity of Lactobacillus detected by real-time quantitative PCR (qPCR); (D) H\&E staining of small intestinal tissue (200x); (E,F) the expression of $\mathrm{ZO}-1$ and $\mathrm{E}$-cadherin in mall intestinal tissues detected by $\mathrm{qPCR} .{ }^{*}, \mathrm{P}<0.05 ;{ }^{* *}, \mathrm{P}<0.01$ vs. CG; ${ }^{+}, \mathrm{P}<0.05,{ }^{++}, \mathrm{P}<0.01$ and ${ }^{+++}, \mathrm{P}<0.001$ vs. LPS; ${ }^{\#}, \mathrm{P}<0.05$ and ${ }^{\# \#}, \mathrm{P}<0.01$ vs. CG. LPS, lipopolysaccharide; CG, control group; Lac, Lactobacillus delbrueckii subsp. bulgaricus.

the model group (Figure 1C). As shown in Figure 1D, the small intestinal mucosa and gland structure of the control group and control + Lac group mice were intact, and the intestinal villi were well structured. However, the small intestinal mucosa and gland structure were disordered and exfoliated in the model group. Compared with that in the model group, the treatment group's intestinal structure disorder was significantly improved.

The tight junction proteins ZO-1 and E-cadherin play an important role in maintaining intestinal mucosal integrity. The qPCR results showed that the expression of ZO-1
mRNA and E-cadherin mRNA was significantly decreased in the model group. Compared with that in the model group, the expression of these tight junction proteins in the treatment group was significantly increased (Figure 1E,F). These results showed that Lac could effectively improve depression-like behavior and maintain intestinal integrity.

\section{Lac inbibited LPS-induced TLR4 signaling in the intestinal tract}

Recent studies have shown that the occurrence and 
A

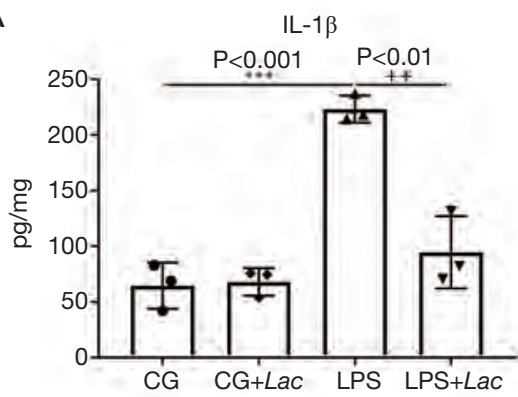

D

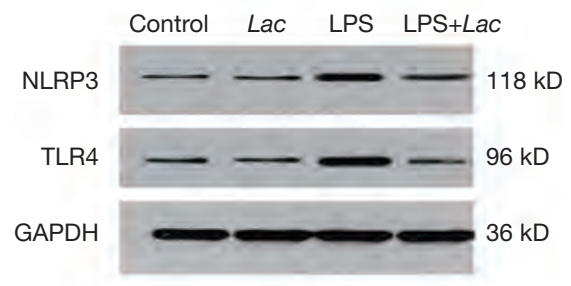

B

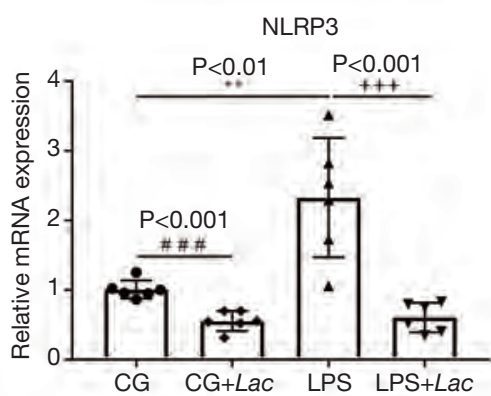

$\mathrm{E}$

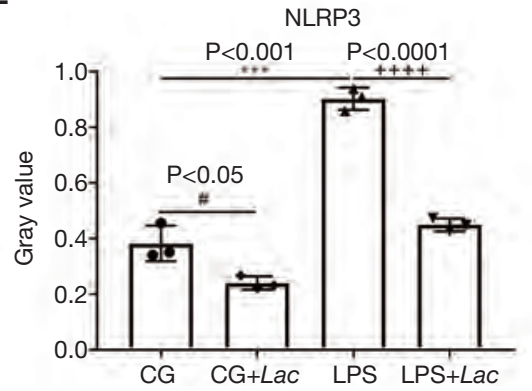

C

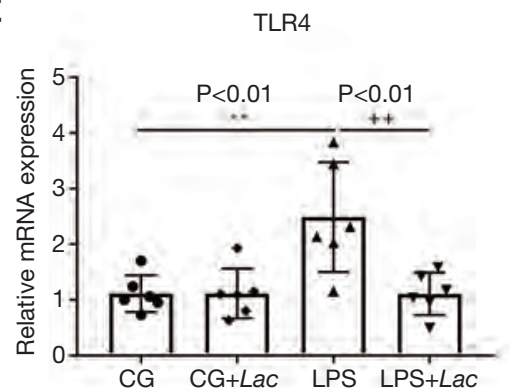

$\mathrm{F}$

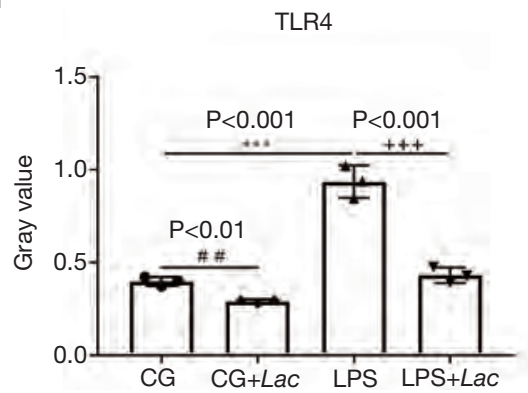

Figure 2 Lac inhibited inflammation in intestinal tract induced by LPS (n=8). (A) The expression level of IL-1 $\beta$ in the small intestine of the mice detected by ELISA; (B,C) the expression of NLRP3 and TLR4 mRNA in the intestinal tract of the mice detected by qPCR; (D) the expression of NLEP3 and TLR4 in intestinal tract of the mice detected by WB; (E,F) gray values of NLRP3 and TLR4 proteins. **, $\mathrm{P}<0.01$ and ${ }^{* * *}, \mathrm{P}<0.001$ vs. CG; ${ }^{++}, \mathrm{P}<0.01{ }^{+++}, \mathrm{P}<0.001$ and $^{++++}, \mathrm{P}<0.0001$ vs. LPS; ${ }^{\#}, \mathrm{P}<0.05,{ }^{\# \#}, \mathrm{P}<0.01$ and ${ }^{\# \# \#}, \mathrm{P}<0.001$ vs. CG. IL- $1 \beta$, interleukin-1 beta; NLRP3, nod-like receptor protein-3; TLR4, toll-like receptor 4; LPS, lipopolysaccharide; CG, control group; Lac, Lactobacillus delbrueckii subsp. bulgaricus.

development of neurological diseases, including depression, are closely related to TLR4 signaling. To explore whether the antidepressant function of Lac is related to TLR4 signaling, we performed tests on the intestinal tract of the mice. The results showed that the level of intestinal IL-1 $\beta$ in the model group was significantly higher than that in the control group. Meanwhile, the level of intestinal IL- $1 \beta$ in the treatment group was significantly lower than that in the model group (Figure $2 A$ ). The results of qPCR showed that the expression of NLRP3 mRNA was significantly increased in the model group, but it was significantly decreased in the treatment group (Figure 2B); the same results were observed for the expression of TLR4 mRNA (Figure 2C). The results of $\mathrm{WB}$ also revealed that the expression of NLRP3 protein was significantly increased in the model group but significantly decreased in the treatment group (Figure 2D,E), and similar observations were made regarding the expression of TLR4 protein (Figure 2D,F). These results indicated that LPS accelerated small intestinal tissues' inflammation by acting on TLR4, but Lac could significantly slow down this process.

\section{Lac significantly improved pathological changes and inflammation in brain tissue in depressed mice}

The qPCR and WB results showed that the mRNA and protein expression levels of microglia marker Iba-1 in the hippocampal tissues of the mice in the model group were significantly higher than those in the control mice, indicating that microglia were in a state of overactivation. After Lac therapy, the levels of mRNA and protein expression of microglia marker Iba-1 were significantly reduced. Meanwhile, the mRNA and protein expression levels of DAT in the model group were significantly lower than those in the control group and were significantly increased after Lac therapy (Figure $3 A, B, C, D, E$ ).

From the ELISA results, we found that the level of IL- $1 \beta$ in the hippocampal tissues of the model group was significantly higher than that in the control group tissues. Meanwhile, in the treatment group, the level of IL- $1 \beta$ was significantly lower than that in the model group (Figure $3 F$ ). The mRNA and protein expression of NLRP 3 was significantly increased in the model group when compared 
A

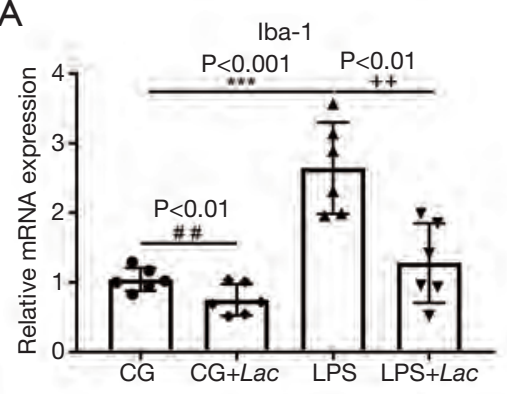

D

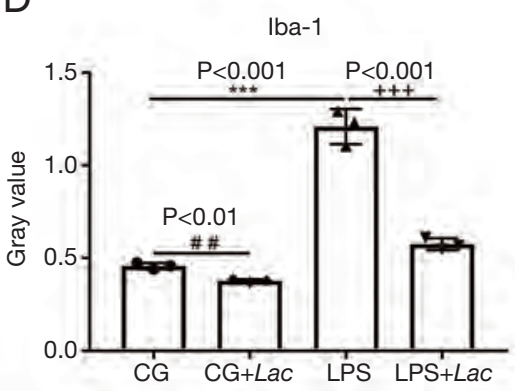

G

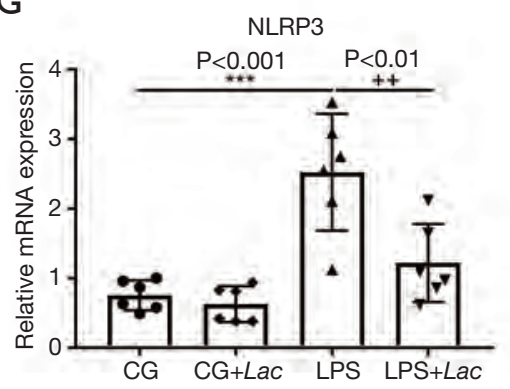

J

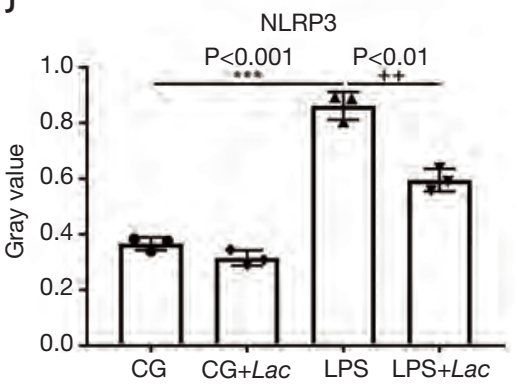

B

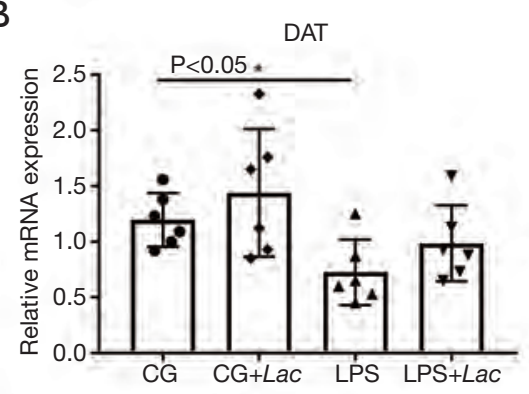

E

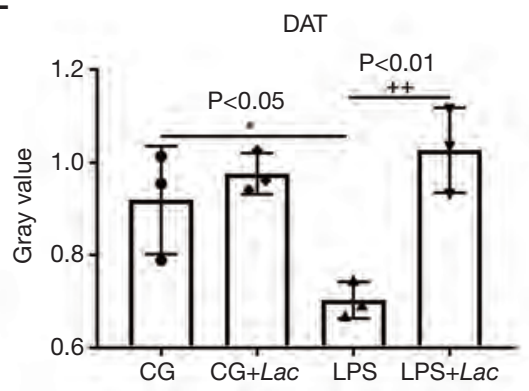

$\mathrm{H}$

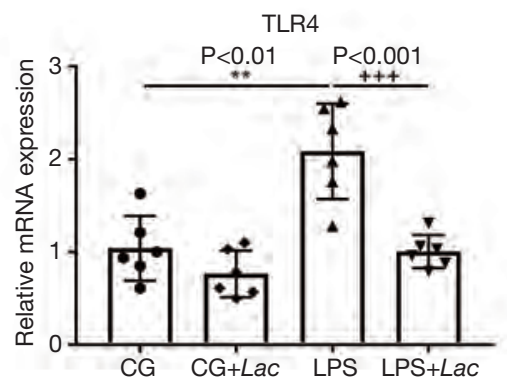

K

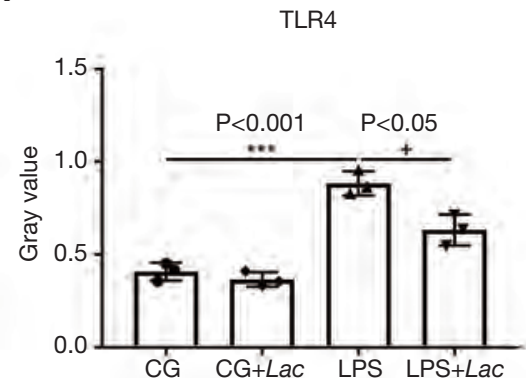

C

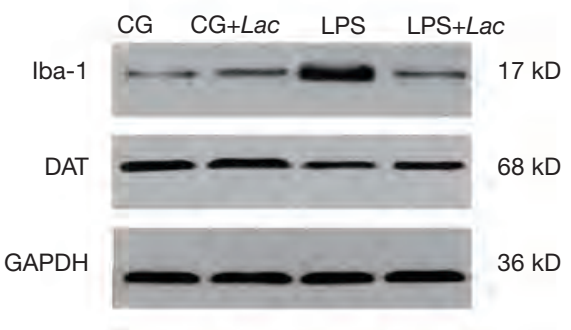

$\mathrm{F}$

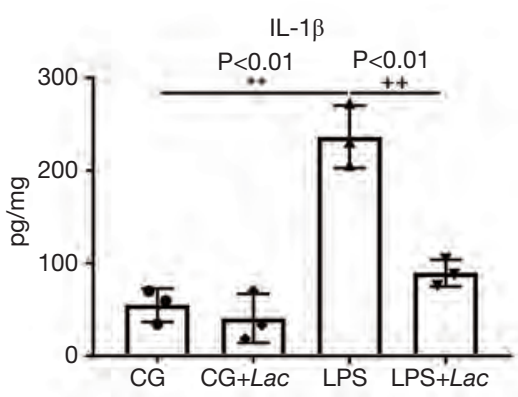

I

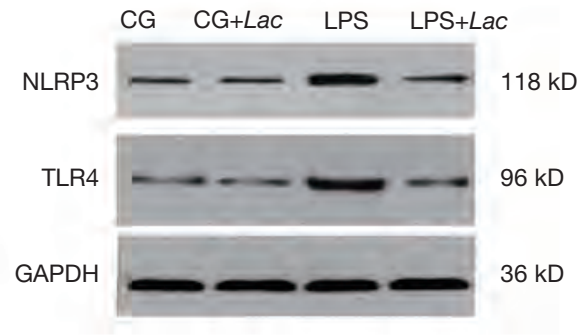

Figure 3 Lac significantly improved the pathological changes in brain tissue and inhibited inflammation in depressed mice $(\mathrm{n}=8)$. $(\mathrm{A}, \mathrm{B}) \mathrm{The}$ expression levels of Iba-1 and DAT mRNA in hippocampal tissues detected by qPCR; (C) the expression of Iba-1 and DAT proteins in hippocampal tissues detected by WB; (D,E) gray values of Iba-1 and DAT proteins; (F) the level of IL-1 $\beta$ in hippocampal tissues detected by ELISA; (G,H) the expression of NLRP3 and TLR4 mRNA in hippocampal tissues detected by qPCR; (I) the expression of NLRP3 and TLR4 proteins in hippocampal tissues detected by WB; $(\mathrm{J}, \mathrm{K})$ gray values of NLRP3 and TLR4 proteins. *, $\mathrm{P}<0.05,{ }^{* *}, \mathrm{P}<0.01$ and ${ }^{* * *}$, $\mathrm{P}<0.001$ vs. $\mathrm{CG}^{+}{ }^{+}, \mathrm{P}<0.05,{ }^{++}, \mathrm{P}<0.01$ and ${ }^{+++}, \mathrm{P}<0.001$ vs. LPS; ${ }^{\text {\#\# }}, \mathrm{P}<0.01$ vs. CG. Iba-1, ionized calcium binding adaptor molecule-1; DAT, dopamine transporter; NLRP3, nod-like receptor protein-3; TLR4, toll-like receptor 4; LPS, lipopolysaccharide; CG, control group; IL-1ß, interleukin-1 beta; WB, Western blot; Lac, Lactobacillus delbrueckii subsp. bulgaricus. 
with the Control group but was significantly decreased in the treatment group compared to in the model group. The mRNA and protein expression of TLR4 showed the same trend as NLRP3 (Figure 3G,H,I,F,K). The results showed that $L a c$ significantly inhibited the over-activation of microglia in brain tissue, promoted dopamine neurons' growth, and reduced inflammation in the brain.

\section{Lac inbibited dysbiosis through its metabolites}

The growth curves of the S. aureus, P. aeruginosa, and Lac in the mixed nutrient broth with different concentrations of MRS were detected to determine the most suitable medium for co-culture (Figure S1A,B,C). The nutrient broth with $20 \%$ MRS was selected as the culture medium for Lac and other common clinical pathogens in the subsequent experiments. Next, we observed the inhibitory effects of $L a c$ on $P$. aeruginosa. The growth of $P$. aeruginosa was completely inhibited when the number of $L a c$ was $\geq 3$ times that of P. aeruginosa (Figure S1D). When the number of Lac was $\geq 3$ times that of other clinical pathogens, Lac's significant inhibitory effects were demonstrated in the normal environment and anaerobic environment (Figure S1E).

The Oxford cup antimicrobial susceptibility test was used to study Lac's inhibitory effects and its fermentation products on common depression-related bacteria. The inhibitory effect of 48-hour Lac fermentation products was stronger than that of 24-hour fermentation products on $S$. epidermidis, $K$. pneumoniae, and $S$. aureus. No difference was found with E. coli, P. aeruginosa, or A. baumanii (Figure 4A). Moreover, the inhibitory effects of 48-hour fermentation products of $L a c$ with or without bacteria were also studied. Cups no. 1 and no. 2 had antibacterial zones, but cups no. 3-5 did not, indicating that $L a c$ inhibited depression-related bacteria through its metabolites (Figure $4 B$ ).

\section{Discussion}

In the past, depression treatment was mainly focused on heredity, behavior, and neurology. In recent years, increasing attention has been paid to the intestinal flora's role, which participates in the bidirectional regulation of the gut and brain through neural, immune, endocrine, and metabolic pathways $(27,28)$. Therefore, probiotics (mainly lactic acid bacteria) with improved intestinal flora functions have attracted more and more attention in this field. Studies have shown that taking lactic acid bacteria can directly or indirectly affect mucosal immunity, improve the intestinal microenvironment, regulate the proportion of bacteria in the intestinal tract, and relieve anxiety, depression, autism, and other mental diseases $(29,30)$. However, the mechanism underlying Lactobacillus' effect in relieving depression and other mental diseases is still unclear.

Our animal experiments showed that Lac could effectively inhibit the production of depression-like behavior in mice. Also, the expression levels of ZO-1 and $\mathrm{E}$-cadherin in the small intestine in the model group were significantly decreased. In contrast, those in the Lac treatment group were significantly increased, suggesting that Lac can effectively ameliorate intestinal mucosal damage in mice with depression-like behavior. Moreover, in mouse hippocampal tissues, overactivation of microglia and decreased expression of DAT, which are closely related to depression, were abrogated by Lac treatment.

Injection of LPS is a commonly used method to establish animal models of depressive behavior. LPS, as an immunoactivator and cytokine inducer, can activate the innate immune system and induce the rapid release of inflammatory cytokines. These inflammatory cytokines act on the brain indirectly or directly, triggering a series of behavioral responses similar to human depression. Consequently, the LPS model has become one of the most commonly used models in studying the role of inflammation in the pathogenesis of depression (31). Our animal experiments showed that the number of $L a c$ in the intestinal tract of LPS-treated mice decreased significantly; the expression levels of TLR4 and NLRP3 in the small intestinal and hippocampal tissues were significantly increased, as was the level of IL-1 $\beta$. These results indicated that LPS accelerated the process of intestinal inflammation and subsequent inflammation in brain tissue via the microbial-intestinal-brain axis, resulting in depressionlike behavior. The expression of TLR4 and NLRP3 and the level of IL- $1 \beta$ in the intestine and brain of mice treated with $L a c$ was significantly decreased, suggesting that $L a c$ treatment can significantly slow the process of inflammation (Figure 5).

After dysbiosis, E. coli, P. aeruginosa, K. pneumoniae, $K$. oxytoca, S. fonticola, S. marcescens, E. aerogenes, A. baumannii, C. freundii, E. cloacae, S. maltophilia, S. aureus, and $S$. epidermidis is often increased, which induces the expression of TLR4. The results of bacteriostasis in vitro showed that the anti-bacterial components of $L a c$ were its fermentation products. The metabolites of $L a c$ include short-chain fatty acids (32), which can interfere with the membrane protein potential and the enzyme activity on the membrane surface 


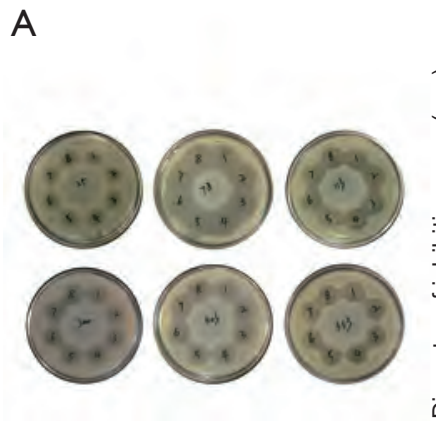

B

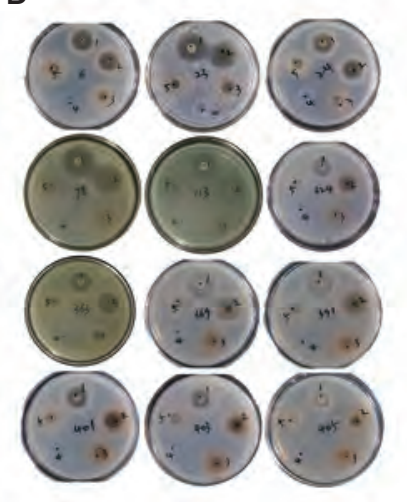

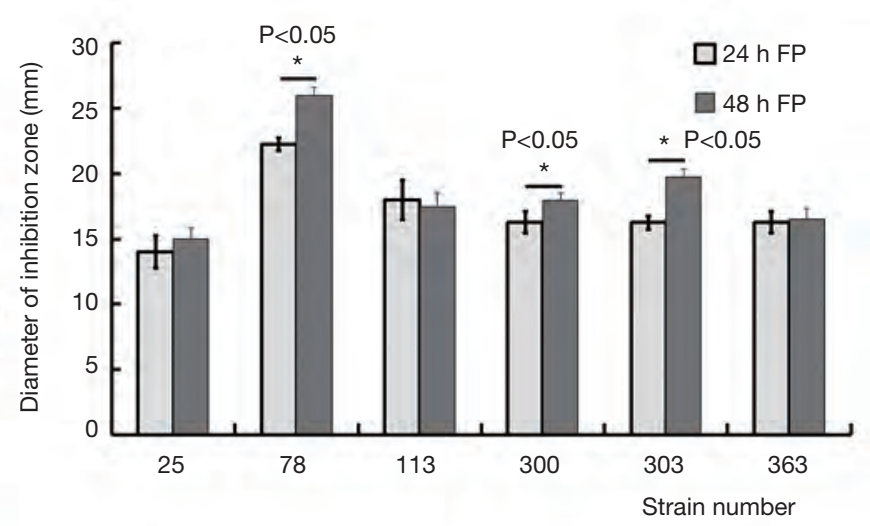

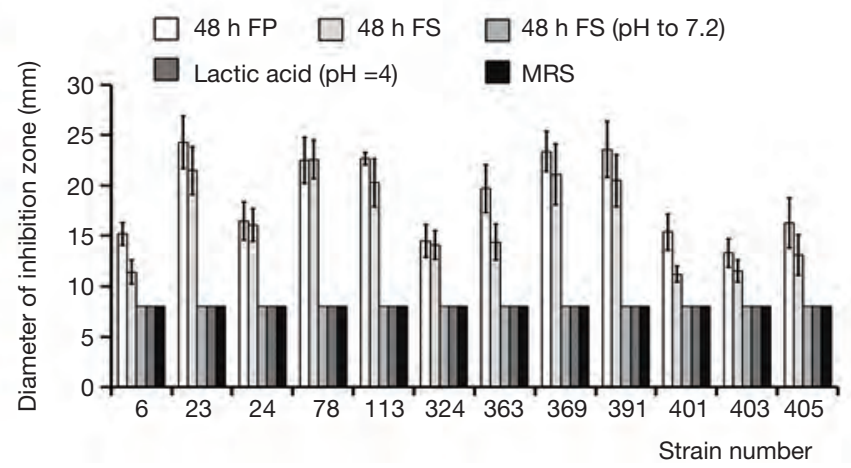

Figure 4 The target bacteria $\left(1.5 \times 10^{7} / \mathrm{mL}\right)$ were coated onto the nutrient agar plate, then 8 Oxford cups were placed on the plate with the supernatant of 24-hour (No. 1-4) and 48-hour (No. 5-6) Lac (200 $\mathrm{LL}$ for each cup). The diameters of the inhibitory zones were measured after incubation at $37^{\circ} \mathrm{C}$ for 24 hours (A). Then, Oxford cups numbered 1, 2, 3, 4, and 5, respectively, were placed on the plate with 48hour FPs in No. 1, 48-hour FS in No. 2, 48-hour FS ( $\mathrm{pH}=7.2)$ in No. 3, lactic acid ( $\mathrm{pH}=4.0)$ in No. 4, and MRS in No. 5 as a control. The diameter of the inhibitory zone was measured after incubation at $37^{\circ} \mathrm{C}$ for 24 hours (B). Number of strains: S. Aureus, 6 and 303 ; MRSA, 23; E. coli, 24 and 25; S. epidermidis, 78; P. aeruginosa, 113; K. pneumoniae, 300; S. fonticola, 324; A. baumanii, 363; C. freumdii, 369; S. marcescens, 401; E. cloacae, 403; S. maltophilia, 405. FP, fermentation products; FS, the supernatant of FP; Lac, Lactobacillus delbrueckii subsp. Bulgaricus.

of microorganisms, thus inhibiting other bacteria's growth and reproduction (33). Undissociated short-chain fatty acids are lipophilic and can bind with phospholipids and LPS on the bacteria's cell membrane, lowering the stability of the bacterial membrane. Undissociated organic acids can even enter into the cytoplasm through the phospholipid bilayer of microorganisms and release $\mathrm{H}^{+}$to alter microorganisms' original $\mathrm{pH}$ environment, resulting in a decrease in enzymatic activity and metabolic disorder of other bacteria $(34,35)$.

Furthermore, Lac can produce bacteriocin (nisin), mainly responsible for changes in the bacterial membrane (36). In the present study, we found Lac could inhibit opportunistic pathogens through their metabolites, therefore inhibiting the expression of pattern recognition receptor TLR4 and subsequent neuroinflammation. However, the specific metabolites of $L a c$ need to be further explored.

LPS is a common bacterial metabolite found in mice and humans that can cause inflammation. Lactobacilli are essential probiotics for the human body. Therefore, this study's results reflect the pathological changes in the human body and provide new ideas and evidence for the alleviation of the symptoms of depression in humans. LPS can also be used in the study of rats and other mammals.

\section{Conclusions}

In conclusion, our study has demonstrated that Lactobacillus metabolites can alleviate depression-like behaviors in mice through inhibiting intestinal inflammation and subsequent 


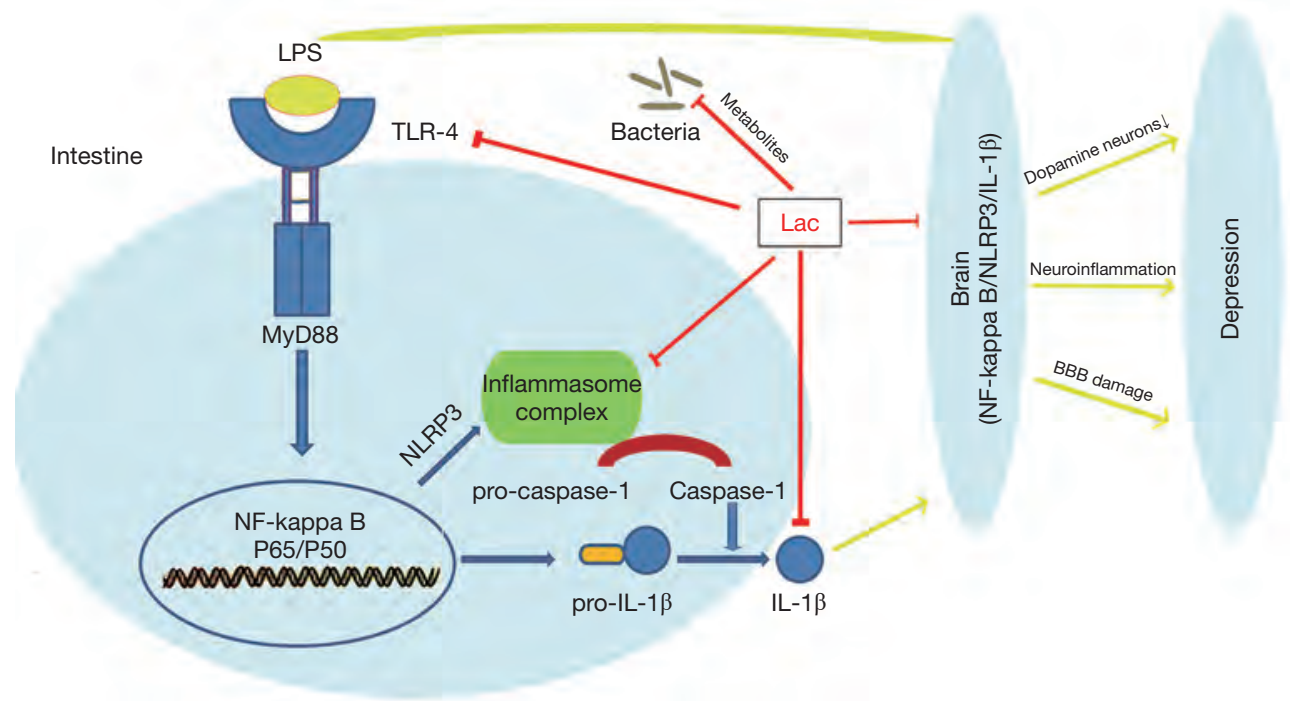

Figure 5 Mechanism of action of Lac. LPS, lipopolysaccharide; TLR4, toll-like receptor 4; Lac, Lactobacillus delbrueckii subsp. bulgaricus; NLRP3, nod-like receptor protein-3; IL-1 $\beta$, interleukin-1 beta.

neuroinflammation. This study has important value for the treatment of patients with depression and the development of antidepressant drugs. It also has important application value for other clinical diseases caused by dysbiosis.

\section{Acknowledgments}

Funding: This work was supported by the National Natural Science Foundation of China (grant No. 31670121 and $31771277)$. The funder had no role in the design or conduct of the study.

\section{Footnote}

Reporting Checklist: The authors have completed the ARRIVE reporting checklist. Available at http://dx.doi. org/10.21037/atm-20-4411

Data Sharing Statement: Available at http://dx.doi. org/10.21037/atm-20-4411

Peer Review File: Available at http://dx.doi.org/10.21037/ atm-20-4411

Conflicts of Interest: All authors have completed the ICMJE uniform disclosure form (available at http://dx.doi. org/10.21037/atm-20-4411). The authors have no conflicts of interest to declare.

Ethical Statement: All animal experiments were performed under a project license (NO.: 2017-S000) granted by the Institutional Ethics Committee of Xiangya School of Medicine, Central South University, in compliance with national or institutional guidelines for the care and use of animals. The authors are accountable for all aspects of the work in ensuring that questions related to the accuracy or integrity of any part of the work are appropriately investigated and resolved.

Open Access Statement: This is an Open Access article distributed in accordance with the Creative Commons Attribution-NonCommercial-NoDerivs 4.0 International License (CC BY-NC-ND 4.0), which permits the noncommercial replication and distribution of the article with the strict proviso that no changes or edits are made and the original work is properly cited (including links to both the formal publication through the relevant DOI and the license). See: https://creativecommons.org/licenses/by-nc-nd/4.0/.

\section{References}

1. Dinan TG, Quigley EM. Probiotics in the treatment of depression: science or science fiction? Aust N Z J Psychiatry 2011;45:1023-5. 
2. Slyepchenko A, Carvalho AF, Cha DS, et al. Gut emotions - mechanisms of action of probiotics as novel therapeutic targets for depression and anxiety disorders. CNS Neurol Disord Drug Targets 2014;13:1770-86.

3. Ménard C, Hodes G E, Russo SJ. Pathogenesis of depression: insights from human and rodent studies. Neuroscience 2016;321:138-62.

4. Hankin BL, Young JF, Abela JRZ, et al. Depression from childhood into late adolescence: Influence of gender, development, genetic susceptibility, and peer stress. J Abnorm Psychol 2015;124:803-16.

5. Petralia MC, Mazzon E, Mangano K, et al. Transcriptomic analysis reveals moderate modulation of macrophage migration inhibitory factor superfamily genes in alcohol use disorders. Exp Ther Med 2020;19:1755-62.

6. Petralia MC, Mazzon E, Fagone P, et al. Retrospective follow-up analysis of the transcriptomic patterns of cytokines, cytokine receptors and chemokines at preconception and during pregnancy, in women with postpartum depression. Exp Ther Med 2019;18:2055-62.

7. Stein DJ, Vasconcelos MF, Albrechet-Souza L, et al. Microglial Over-Activation by Social Defeat Stress Contributes to Anxiety- and Depressive-Like Behaviors. Front Behav Neurosci 2017;11:207.

8. Brites D, Fernandes A. Neuroinflammation and Depression: Microglia Activation, Extracellular Microvesicles and microRNA Dysregulation. Front Cell Neurosci 2015;9:476.

9. Zhao X, Cao F, Liu Q, et al. Behavioral, inflammatory and neurochemical disturbances in LPS and UCMSinduced mouse models of depression. Behav Brain Res 2019;364:494-502.

10. Yirmiya R, Rimmerman N, Reshef R. Depression as a microglial disease. Trends Neurosci 2015;38:637-58.

11. Murray E, Sharma R, Smith KB, et al. Probiotic consumption during puberty mitigates LPS-induced immune responses and protects against stress-induced depression- and anxiety-like behaviors in adulthood in a sex-specific manner. Brain Behav Immun 2019;81:198-212.

12. Li M, Li C, Yu H, et al. Lentivirus-mediated interleukin$1 \beta$ (IL-1 $\beta$ ) knock-down in the hippocampus alleviates lipopolysaccharide (LPS)-induced memory deficits and anxiety- and depression-like behaviors in mice. J Neuroinflammation 2017;14:190.

13. Cepeda MS, Katz EG, Blacketer C. Microbiome-Gut-Brain Axis: Probiotics and Their Association with Depression. J Neuropsychiatry Clin Neurosci 2017;29:39-44.

14. Malick M, Gilbert K, Daniel J, et al. Vagotomy prevents the effect of probiotics on caspase activity in a model of postmyocardial infarction depression. Neurogastroenterol Motil 2015;27:663-71.

15. Westfall E, Brandenburg D. Probiotics for Symptoms of Depression and Anxiety. Am Fam Physician 2018;97:Online.

16. Zheng P, Zeng B, Zhou C, et al. Gut microbiome remodeling induces depressive-like behaviors through a pathway mediated by the host's metabolism. Mol Psychiatry 2016;21:786-96.

17. Arseneault-Bréard J, Rondeau I, Gilbert K, et al. Combination of Lactobacillus helveticus R0052 and Bifidobacterium longum R0175 reduces post-myocardial infarction depression symptoms and restores intestinal permeability in a rat model. Br J Nutr 2012;107:1793-9.

18. Akkasheh G, Kashani-Poor Z, Tajabadi-Ebrahimi M, et al. Clinical and metabolic response to probiotic administration in patients with major depressive disorder: A randomized, double-blind, placebo-controlled trial. Nutrition 2016;32:315-20.

19. Borrelli L, Aceto S, Agnisola C, at al. Probiotic modulation of the microbiota-gut-brain axis and behaviour in zebrafish. Sci Rep 2016;6:30046.

20. Abildgaard A, Elfving B, Hokland M, et al. Probiotic treatment reduces depressive-like behaviour in rats independently of diet. Psychoneuroendocrinology 2017;79:40-8.

21. Bajinka O, Tan Y, Abdelhalim KA, et al. Extrinsic factors influencing gut microbes, the immediate consequences and restoring eubiosis. AMB Express 2020;10:130.

22. Yin X, Salemi MR, Phinney BS, et al. Proteomes of Lactobacillus delbrueckii subsp. bulgaricus LBB.B5 Incubated in Milk at Optimal and Low Temperatures. mSystems 2017;2:e0027-17.

23. Usui Y, Kimura Y, Satoh T, et al. Effects of long-term intake of a yogurt fermented with Lactobacillus delbrueckii subsp. bulgaricus 2038 and Streptococcus thermophilus 1131 on mice. Int Immunol 2018;30:319-31.

24. Qiu X, Ye Q, Sun M, et al. Saturated hydrogen improves lipid metabolism disorders and dysbacteriosis induced by a high-fat diet. Exp Biol Med (Maywood) 2020;245:512-21.

25. Li L, Liu M, Kang L, et al. HHEX: A Crosstalker between HCMV Infection and Proliferation of VSMCs. Front Cell Infect Microbiol 2016;6:169.

26. Qin L, Qiu K, Hu C, et al. Respiratory syncytial virus promoted the differentiation of Th17 cells in airway microenvironment through activation of Notch-1/Delta3. J Med Microbiol 2019;68:649-56. 


\section{Page 12 of 12}

27. Dinan TG, Cryan JF. Gut-brain axis in 2016: Brain-gutmicrobiota axis-mood, metabolism and behaviour. Nat Rev Gastroenterol Hepatol 2017;14:69-70.

28. Evrensel A, Ceylan ME. The Gut-Brain Axis: The Missing Link in Depression. Clin Psychopharmacol Neurosci 2015;13:239-44.

29. Gareau MG. Cognitive Function and the Microbiome. Int Rev Neurobiol 2016;131:227-46.

30. Wang H, Lee IS, Braun C, et al. Effect of Probiotics on Central Nervous System Functions in Animals and Humans: A Systematic Review. J Neurogastroenterol Motil 2016;22:589-605.

31. Guo LT, Wang SQ, Su J, et al. Baicalin ameliorates neuroinflammation-induced depressive-like behavior through inhibition of toll-like receptor 4 expression via the PI3K/AKT/FoxO1 pathway. J Neuroinflammation 2019; $16: 95$.

Cite this article as: Qiu X, Wu G, Wang L, Tan Y, Song Z. Lactobacillus delbrueckii alleviates depression-like behavior through inhibiting toll-like receptor 4 (TLR4) signaling in mice. Ann Transl Med 2021;9(5):366. doi: 10.21037/atm-20-4411

\section{Qiu et al. Lactobacillus alleviates depression-like behavior}

32. Callon C, Arliguie C, Montel MC. Control of Shigatoxinproducing Escherichia coli in cheese by dairy bacterial strains. Food Microbiol 2016;53:63-70.

33. Stoianova LG, Ustiugova EA, Netrusov AI. Antibacterial metabolites of lactic acid bacteria: their diversity and properties. Prikl Biokhim Mikrobiol 2012;48:259-75.

34. Gong Y, Li T, Li S, et al. Achieving high yield of lactic acid for antimicrobial characterization in cephalosporinresistant lactobacillus by the co-expression of the phosphofructokinase and glucokinase. J Microbiol Biotechnol 2016;26:1148-61.

35. Wales A, McLaren I, Rabie A, et al. Assessment of the anti-Salmonella activity of commercial formulations of organic acid products. Avian Pathol 2013;42:268-75.

36. Lee SY, Kang DH. Survival mechanism of Escherichia coli O157:H7 against combined treatment with acetic acid and sodium chloride. Food Microbiol 2016;5 5:95-104. 
A
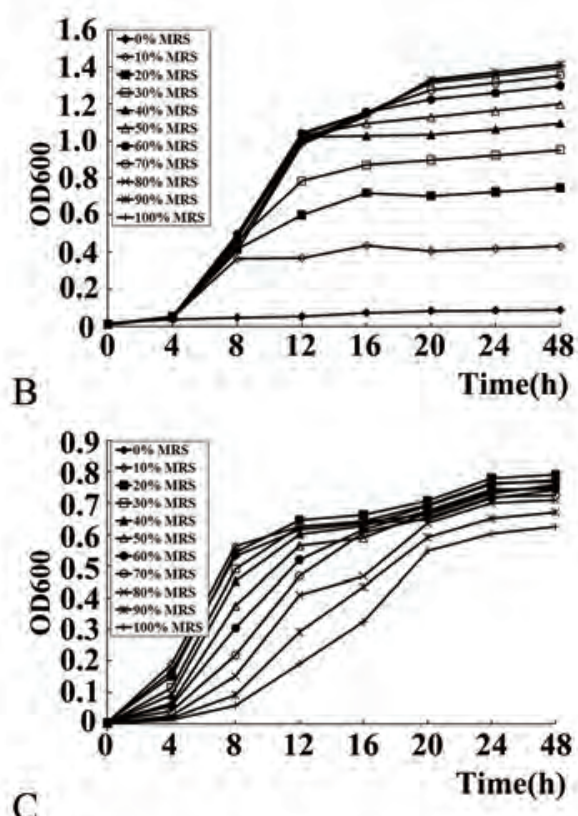

C

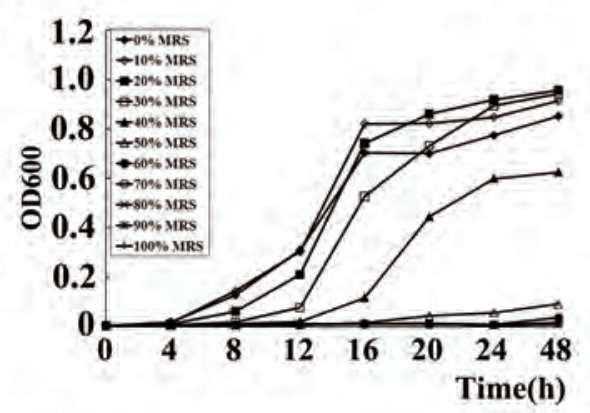

D
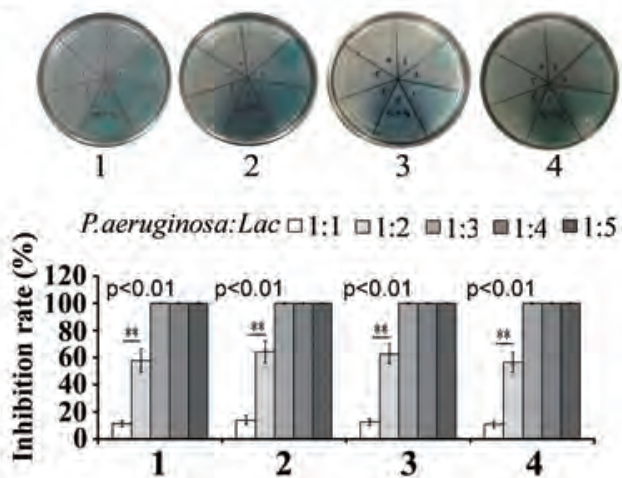

E
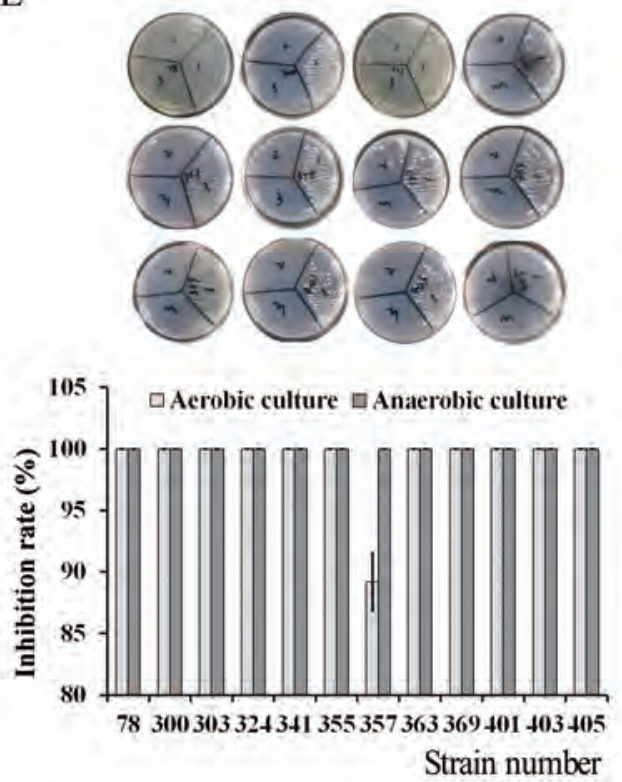

Figure S1 Growth curves of Lac (A), S. aureus (B), and P. aeruginosa (C) in nutrient broth with different proportion of MRS. (D) P. aeruginosa and Lac were mixed at a proportions of 1:1, 1:2, 1:3, 1:4, and 1:5, and then inoculated in nutrient broth with 20\% MRS. 1, Co-culture under anaerobic conditions and the tested bacteria after co-culture under aerobic conditions; 2, co-culture and the tested bacteria after coculture under anaerobic conditions; 3, co-culture and the tested bacteria after co-culture under aerobic conditions; 4, co-culture under aerobic conditions and the tested bacteria after co-culture under anaerobic conditions. (E) The tested bacteria and Lac (1:3) were mixed and co-cultured in nutrient broth with 20\% MRS and incubated under aerobic or anaerobic conditions, respectively. Number of strains: $S$. epidermidis, 78; K. pneumoniae, 300; S. aureus, 303; S. fonticola, 324; P. aeruginosa, 341; E. aerogenes, 355; K. oxytoca, 357; A. baumanii, 363; C. freumdii, 369; S. marcescens, 401; E. cloacae, 403; S. maltophilia, 405. 
Table S1 Primers for quantitative polymerase chain reaction

\begin{tabular}{|c|c|c|}
\hline Gene name & Sequence & Product length (bp) \\
\hline E-cadherin & $\begin{array}{l}\text { F: 5'-CTGGGATGGAGGTAAAGTAAGCA-3' } \\
\text { R: 5'-TCGGGATGAGCGGATAGCGT-3' }\end{array}$ & 154 \\
\hline$N L R P 3$ & $\begin{array}{l}\text { F: 5'-CCACCCTGAGTAGATGCTGTTTA-3' } \\
\text { R: 5'-TGGGATGTTCTGTTTGCTGTT-3' }\end{array}$ & 121 \\
\hline TLR4 & $\begin{array}{l}\text { F: 5'-ACACCTGCСTCTTCССTCCC-3' } \\
\text { R: 5'-CTCCAGTCGGTCAGCAAACG-3' }\end{array}$ & 185 \\
\hline$D A T$ & $\begin{array}{l}\text { F: 5'-GCGTCCCACTTGCTTTGTCTC-3' } \\
\text { R: 5'-CAAGTCTCCGTCCCTGTTCG-3' }\end{array}$ & 274 \\
\hline
\end{tabular}

